\title{
Kinetic Approaches to Measuring Peroxiredoxin Reactivity
}

\author{
Christine C. Winterbourn*, and Alexander V. Peskin
}

\begin{abstract}
Peroxiredoxins are ubiquitous thiol proteins that catalyse the breakdown of peroxides and regulate redox activity in the cell. Kinetic analysis of their reactions is required in order to identify substrate preferences, to understand how molecular structure affects activity and to establish their physiological functions. Various approaches can be taken, including the measurement of rates of individual steps in the reaction pathway by stopped flow or competitive kinetics, classical enzymatic analysis and measurement of peroxidase activity. Each methodology has its strengths and they can often give complementary information. However, it is important to understand the experimental conditions of the assay so as to interpret correctly what parameter is being measured. This brief review discusses different kinetic approaches and the information that can be obtained from them.
\end{abstract}

\section{INTRODUCTION}

Peroxiredoxins (Prxs) are now well established as important players in redox biology. They were initially described as antioxidant proteins (Rhee et al., 2005). However, as the redox biology field has evolved, and it has become appreciated that oxidation and reduction reactions play a fundamental role in regulating physiological activity and pathological responses in cells, it is more instructive to consider their function within the wider spectrum of redox regulatory activity. In some situations this could be synonymous with antioxidant (or oxidant scavenging) activity, but in others, the action of a Prx is better considered as reacting with an oxidant either to restrict its reaction with another target, or to generate an intermediate that can transmit oxidizing equivalents to another target by a redox relay mechanism (Fig. 1) (Winterbourn and Hampton, 2015).

Prxs are thiol proteins that are notable for their high reactivity and specificity for peroxides (Karplus, 2015). Early studies

Centre for Free Radical Research, Department of Pathology, University of Otago, Christchurch, New Zealand

*Correspondence: christine.winterbourn@otago.ac.nz

Received 30 November, 2015; accepted 3 December, 2015; published online 25 January, 2016

Keywords: hyperoxidation, kinetics, peroxide, peroxiredoxin, thiol oxidation demonstrated surprisingly low reactivity with thiol reagents such as iodoacetamide and other oxidants such as chloramines (Peskin et al., 2007) and it is clear that the low $\mathrm{pK}_{\mathrm{a}}$ of the active site thiol is insufficient to confer the high peroxide reactivity. In fact, typical low molecular weight and protein thiolates react with $\mathrm{H}_{2} \mathrm{O}_{2}$ with a rate constant of $20 \mathrm{M}^{-1} \mathrm{~s}^{-1}$ whereas values of Prxs are $10^{5}-10^{6}$ fold higher (Winterbourn and Hampton, 2008). An elegant series of structural and mutational studies (Hall et al., 2010; Nakamura et al., 2010; Nagy et al., 2011) have shown that to get sufficient rate enhancement, it is necessary to activate the peroxide. As discussed in detail elsewhere (Hall et al., 2010 ; 2011), this involves formation of a transition state in which hydrogen bonding of the peroxide to conserved Arg and Thr residues and an amide nitrogen in the active site facilitates breakage of its -O-O- bond and oxidation of the thiolate to a sulfenic acid. This mechanism operates with $\mathrm{H}_{2} \mathrm{O}_{2}$, peroxynitrite (which also has a peroxyl motif) and organic peroxides such as derived from cumene, linoleic or amino acids. Individual Prxs vary in their preference for different peroxides, although it is not fully understood how their structural differences dictate this selectivity (Karplus, 2015)

Once formed, the sulfenic acid condenses with another thiol (for typical 2-Cys Prxs, the resolving Cys on the opposing subunit of the functional homodimer) to give a disulfide (Fig. 2). The disulfide is then reduced to complete the redox cycle. This step can be accomplished by thioredoxin/thioredoxin reductase, which are considered to be the main physiological mechanism, but DTT and thiol proteins containing a thioredoxin motif can also act as reductants (Karplus, 2015; Rhee et al., 2005). Reaction of the sulfenic acid with GSH and reduction of the mixed disulfide with glutaredoxin also provides an alternative recycling mechanism (Peskin et al., 2016).

The sulfenic acid can be further oxidized by a second peroxide to form a sulfinic acid, a reaction referred to as hyperoxidation. This reaction competes with disulfide formation and occurs more readily when disulfide formation is slow. Disulfide formation requires a structural rearrangement from the fully folded (FF) to locally unfolded (LU) conformation, and this occurs at different rates depending on the structure of the C-terminus. It is generally slower in eukaryotic Prxs, which tend to be more sensitive to hyperoxidation than their prokaryotic counterparts (Wood et al., 2003). However, there are variations within the eukaryotic Prxs, with the rate of disulfide formation being almost 10-fold faster for Prx3 than Prx2 and its susceptibility to hyperoxidation being consequently less (Cox et al., 2009a; Peskin et al., 2013). 
1. Antioxidant activity

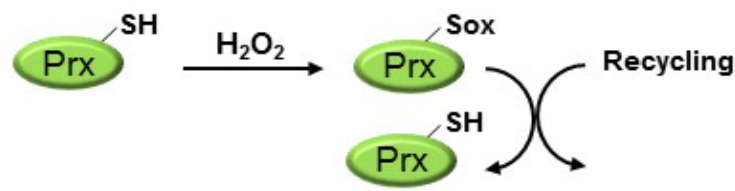

2. Competition with a redox-sensitive target

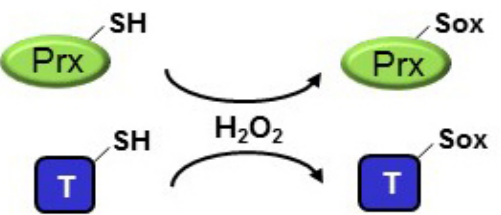

3. Activation of a redox-sensitive target

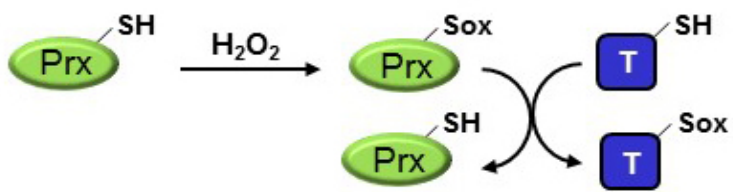

Fig. 1. Possible redox-regulatory mechanisms for peroxiredoxins. $\mathrm{T}$, oxidant-sensitive target.

In order to understand the redox properties of Prxs, and to explain how they function in biological systems where there are competing reactions for peroxides and reductants, it is important to have kinetic data on the rates of their different reactions. There are several ways of obtaining this, each of which may provide different information. This brief article summarizes the different approaches.

\section{COMPETITION KINETICS}

Reactions of Prxs with $\mathrm{H}_{2} \mathrm{O}_{2}$ and most other peroxides are so fast that they need to be studied by rapid reaction kinetic tech- niques such as stopped flow, or by competition with another reaction for which the relevant rate constant is known. The most widely used approach has been competition kinetics. This requires a sensor that reacts at a similar rate to the Prx to give an easily detectable and relatively stable product. A substoichiometric amount of the peroxide is added, the amount of oxidation of the sensor measured, then varying concentrations of the Prx are added and the extent of inhibition determined (Fig. 3A). As first described (Ogusucu et al., 2007), horseradish peroxidase (HRP) has been effectively used for this purpose. Native HRP reacts at an appropriate rate $\left(\mathrm{k}=1.7 \times 10^{7} \mathrm{M}^{-1} \mathrm{~s}^{-1}\right.$ for $\mathrm{H}_{2} \mathrm{O}_{2}$ ) to form spectrally distinct Compound I, which under most conditions is stable for seconds to minutes. Rate constants for reaction 1 in Fig. 2 in the $10^{7} \mathrm{M}^{-1} \mathrm{~s}^{-1}$ range have been measured for a number of Prxs (Cox et al., 2009b; Hugo et al., 2009; Manta et al., 2009; Nagy et al., 2011; Ogusucu et al., 2007; Peskin et al., 2007), as illustrated for Prx3 in Fig. 3B. A similar approach has been used with peroxynitrite and HRP (although this may be complicated by multiple interactions) or with $\mathrm{Mn}$ porphyrins that react at appropriate rates (Hugo et al., 2009; Trujillo et al., 2008).

The HRP assay is really only applicable to Prxs with rate constants of at least $10^{6} \mathrm{M}^{-1} \mathrm{~s}^{-1}$. If the Prx is less reactive, much higher concentrations have to be used and this can be problematic. Compound I of HRP then becomes susceptible to reduction by the high thiol concentration and this gives the false impression that the Prx has higher peroxide reactivity than is actually the case. This is a limitation for some native Prxs and particularly for mutated proteins with decreased reactivity. An alternative approach that has been applied to the Mycobacterium tuberculosis Prx, AhpE (Hugo et al., 2009) is to substitute $\mathrm{HRP}$ for lignin peroxidase, which is 100 -fold less reactive with $\mathrm{H}_{2} \mathrm{O}_{2}$. Rate constants of $\sim 10^{4} \mathrm{M}^{-1} \mathrm{~s}^{-1}$ for reactions of amino acid peroxides with Prxs 2 and 3 have also been determined by measuring peroxide loss by mass spectrometry and quenched flow (Peskin et al., 2010).

Competition with catalase can also be used to assess the $\mathrm{H}_{2} \mathrm{O}_{2}$ reactivity of Prxs (Peskin et al., 2007). This method is best suited to typical 2Cys Prxs, as oxidation can be monitored by blocking with $\mathrm{N}$-ethylmaleimide and detecting dimerization by non-reducing SDS-PAGE (Fig. 3C). The assay is less quantita-

\section{Reduced}
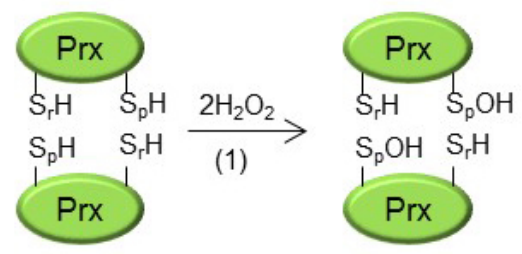

$2 \mathrm{H}_{2} \mathrm{O}_{2}$

(2)

Hyperoxidized monomer

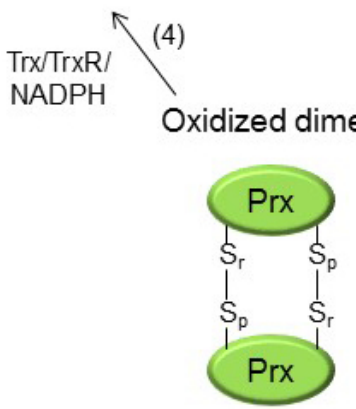

$\mathcal{L}(3) \quad(2 / 3) \mathrm{H}^{\mathrm{H}_{2}}$

Hyperoxidized dimer

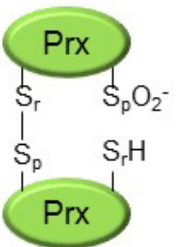

Fig. 2. Redox transformations of a typical 2Cys Prx. Sp and $\mathrm{Sr}$ refer to the peroxidatic and resolving Cys respectively; Trx, thioredoxin; TrxR, thioredoxin reductase. Oxidation at two active sites of the homodimer is shown. Although interaction between the two is possible, in this article we have considered the kinetics of two acting independently. Numbers in parenthesis refer to the reactions shown and are described in the text. 
tive than with HRP, but gives a good visual representation. The rate constant for bovine catalase is less than for Prx2 and this is evident by the intense catalase band required to suppress oxidation.

The rate of reaction of the active site sulfenic acid with a second $\mathrm{H}_{2} \mathrm{O}_{2}$ (hyperoxidation; reaction 2, Fig. 2) and its rate of condensation with the resolving Cys (reaction 3) can also be determined by competitive kinetics (Peskin et al., 2013). The rates of these reactions can be expressed kinetically as

Hyperoxidation $=\mathrm{k}_{2}[\mathrm{PrxSOH}]\left[\mathrm{H}_{2} \mathrm{O}_{2}\right]$

Disulfide formation $=\mathrm{k}_{3}[\mathrm{PrxSOH}]$.

Thus a plot of the ratio of these two products as a function of $\mathrm{H}_{2} \mathrm{O}_{2}$ concentration is linear with a slope corresponding to $\mathrm{k}_{2} / \mathrm{k}_{3}$. Absolute values for the two rate constants can be obtained by carrying out the reaction in the presence of varying concentrations of catalase, which competes with hyperoxidation. This does not give a simple rate equation but the data can be analysed by a kinetic program such as Berkeley Madonna, to obtain rate constants that give the best fit. Using this system we obtained rate constants $\left(\mathrm{k}_{2}\right)$ of $10^{4} \mathrm{M}^{-1} \mathrm{~s}^{-1}$ for hyperoxidation of Prx2 and Prx3. The sulfenic acid is oxidized 1000 times more slowly than the thiolate but is nevertheless several orders of magnitude more reactive than most reduced thiol proteins (Peskin et al., 2013). This study gave a rate constant for condensation of the sulfenic acid $\left(\mathrm{k}_{3}\right)$ of $\sim 2 \mathrm{~s}^{-1}$ for Prx2 and almost 10-fold higher for Prx3, explaining the latter's greater resistance to hyperoxidation. Consistent with this trend, a value of $80 \mathrm{~s}^{-1}$ was determined by stopped flow methodology for the even more resistant bacterial Prx, AhpC (Parsonage et al., 2015).

One of the more challenging aspects of studying reduced Prxs is to achieve efficient reduction of the active site thiol and maintain it in a reduced form while removing excess reducing agent. The Prxs are best stored as disulfides and reduced prior to use. Storage in DTT is best avoided, as autoxidation of the DTT gives rise to $\mathrm{H}_{2} \mathrm{O}_{2}$, enabling ongoing cycling of the Prx with potential for inactivation. Most investigators incubate with DTT (commonly $20 \mathrm{mM}$ over an hour) to reduce the protein. Some but not all Prxs, can be reduced by TCEP (tris-(2-carboxyethyl) phosphine), but even though TCEP conjugated to beads should be ideal for the purpose, we could not achieve efficient reduction using a commercial product. The more difficult task is to remove the reductant without the Prx re-oxidizing. Re-oxidation requires only traces of peroxide in a buffer, especially if the Prx encounters relatively large volumes of buffer such as during passage through a spin column. Traces of $\mathrm{H}_{2} \mathrm{O}_{2}$ are almost invariably present in buffers. Some investigators overcome this

A Principle:A peroxide at a sub-stoichiometric concentration is added to a sensor (in this case HRP) and the extent of inhibition of sensor oxidation by increasing concentrations of the Prx is determined. The kinetic analysis can be simply expressed as follows (where $k_{\text {HFp }}$ and $k_{p \times x}$ refer to the rate constants for reaction of each substrate with $\mathrm{H}_{2} \mathrm{O}_{2}$ ).

$$
\begin{aligned}
& \mathrm{H}_{2} \mathrm{O}_{2}+\mathrm{HRP} \longrightarrow \mathrm{HRP} \mathrm{Cpdl} \quad \text { Rate }=\mathrm{k}_{\text {HFp }}[\mathrm{HRP}] \cdot\left[\mathrm{H}_{2} \mathrm{O}_{2}\right] \\
& \mathrm{H}_{2} \mathrm{O}_{2}+\mathrm{Prx}-\mathrm{SH} \longrightarrow \mathrm{Prx}-\mathrm{SOH} \quad \text { Rate }=\mathrm{k}_{\mathrm{prx}}[\mathrm{Prx}] \cdot\left[\mathrm{H}_{2} \mathrm{O}_{2}\right]
\end{aligned}
$$

The fractional inhibition of HRP oxidation by the $\mathrm{Prx}(\mathrm{F})$ represents the proportion of the $\mathrm{H}_{2} \mathrm{O}_{2}$ reacting with the Prx and this can be expressed kinetically as:

$\mathrm{F} /(1-\mathrm{F})=\mathrm{k}_{\mathrm{pr}}[\mathrm{Prx}] \cdot\left[\mathrm{H}_{2} \mathrm{O}_{2}\right] / \mathrm{K}_{\mathrm{HRP}}[\mathrm{HRP}] \cdot\left[\mathrm{H}_{2} \mathrm{O}_{2}\right]$ $\mathrm{K}_{\mathrm{prx}}$. Is determined from the slope of a plot of $\{(\mathrm{F}) /(1-\mathrm{F})\} . \mathrm{K}_{\mathrm{HFp}} .[\mathrm{HRP}] \vee[\mathrm{PrX}]$
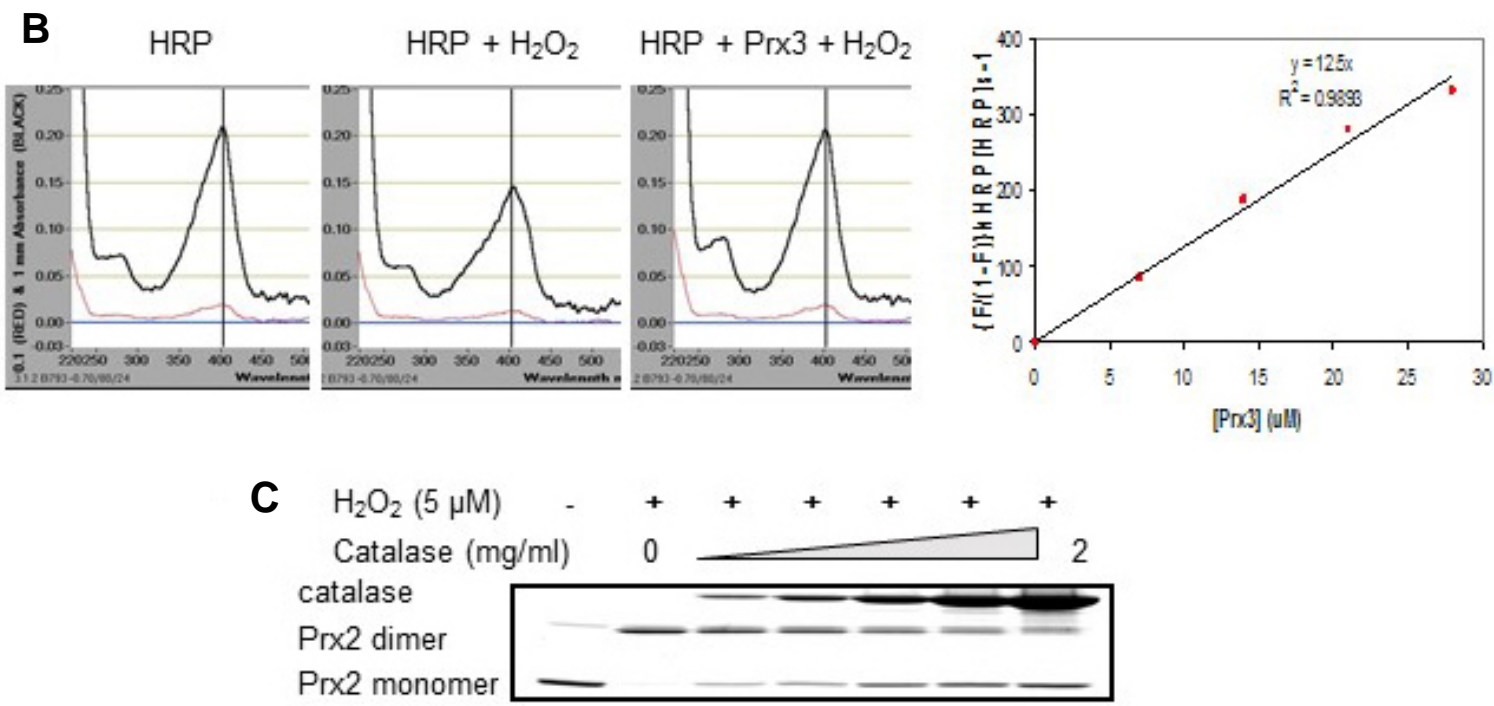

Fig. 3. Competitive kinetics with HRP for measuring Prx rate constants. (A) Principle; (B) Example showing spectral changes and calculation of the rate constant for Prx3. (C) Inhibition of Prx2 oxidation by increasing concentrations of catalase as shown by non-reducing SDS-PAGE. Data are from (Peskin et al., 2007) and (Cox et al., 2009b). 
with extensive de-gassing and argon purging. We prefer to treat buffers with catalase, either enclosed in a dialysis sac, or in solution with subsequent ultrafiltration. We also find a need to pretreat the resin in spin columns with catalase before use (Peskin et al., 2007).

\section{DIRECT MEASUREMENTS BY STOPPED FLOW}

The reaction between a $\mathrm{Prx}$ and $\mathrm{H}_{2} \mathrm{O}_{2}$ or another oxidant can sometimes be studied by stopped flow. This requires the formation of a detectable intermediate or product that can be followed by direct measurement over a millisecond timescale. It normally employs UVNis or fluorimetric detection. It has been applied to a limited extent with Prxs, where changes in fluorescence of Trp residue(s) near the active site have been followed. This was first employed to study the reaction of peroxynitrite and various peroxides with Prx5, an atypical 2-Cys Prx that forms an internal disulfide within a single chain (Trujillo et al., 2007). A difference in Trp fluorescence between the reduced and disulfide forms enabled rates of oxidation to be measured, with rate constants $\left(10^{5}\right.$ to approaching $10^{8} \mathrm{M}^{-1} \mathrm{~s}^{-1}$ for the different oxidants) in good agreement with other methodologies. At that time it was thought that a similar approach was not applicable to other Prxs, as they show no difference in Trp fluorescence between reduced and oxidized forms. However, it has recently been shown with bacterial AhpC that formation of intermediates during oxidation results in transient changes in Trp fluorescence that can be followed by stopped flow (Parsonage et al., 2015). Kinetic analysis was complex but it was possible to distinguish different steps which were attributed to reversible $\mathrm{H}_{2} \mathrm{O}_{2}$ binding, thiolate oxidation to the sulfenic acid, then disulfide formation. This approach may not be as widely applicable as competition assays and it is reassuring that there is general agreement between the two. However, it has the advantage of being able to separate out binding from thiolate oxidation, and in this case identifying the rate of binding as varying between different oxidants.

Fast reaction kinetics can also in principle be used for measuring Prx reduction rates. For example, by following Trp changes in Prx5, Trujillo et al. measured a rate constant for reduction $\left(\mathrm{k}_{4}\right)$ of $\sim 10^{6} \mathrm{M}^{-1} \mathrm{~s}^{-1}$. (Trujillo et al., 2007). Parsonage formed a Trp mutant at the N-terminal domain of AhpF, the specific flavoprotein reductase for $\mathrm{AhpF}$, to measure a rate constant for reduction of $\sim 10^{7} \mathrm{M}^{-1} \mathrm{~s}^{-1}$. (Parsonage et al., 2015). More experimental detail on these techniques and those discussed below is given elsewhere (Nelson and Parsonage, 2011; Trujillo et al., 2008).

\section{CLASSICAL ENZYMOLOGY}

Peroxiredoxins can be studied using classical enzyme kinetic methods, provided there is good understanding of the assay conditions and which step(s) in the reaction are rate-limiting. For example, based on initial enzymatic studies it was concluded that Prxs react too slowly with $\mathrm{H}_{2} \mathrm{O}_{2}$ to compete with glutathione peroxidases and catalase and would have only a minor role in physiological $\mathrm{H}_{2} \mathrm{O}_{2}$ removal. However, these experiments were carried out under conditions where the rate of reduction of the Prx was rate limiting and it was necessary to optimize the reductive steps, as was done with AhpC (Parsonage et al., 2005), to reveal the high Prx reactivity. When this is done, enzymatic parameters agree well with data obtained by direct rate constant measurements (Nelson and Parsonage, 2011).

The enzymology of the Prxs can be studied by measuring the rate of peroxide consumption or the rate of NADPH oxidation in the presence of a recycling system such as thioredoxin/thioredoxin reductase, as detailed in (Nelson and Parsonage, 2011). This type of analysis has established that the reaction proceeds via a ping pong mechanism in which one substrate modifies the enzyme and leaves before the second substrate interacts. It provides values for kinetic constants such as $k_{\text {cat }}$ and $\mathrm{K}_{\mathrm{m}}$ for the different substrates (with $\mathrm{k}_{\mathrm{cat}} / \mathrm{K}_{\mathrm{m}}$ relating to rate constants obtained by direct measurement). However, these data must be interpreted cautiously and not assumed to have the meaning attributed to them by Michaelis/Menton kinetics. Unless the reductive steps are maximized, the $\mathrm{K}_{m}$ for $\mathrm{H}_{2} \mathrm{O}_{2}$ varies with the reductant concentration and reflects turnover rate rather than saturation of $\mathrm{H}_{2} \mathrm{O}_{2}$ binding. Therefore it needs to be established that saturation is achieved. This may be difficult as it requires very high concentrations of each of the substrates in the reduction cycle. For example, DTT can be used as a reductant, but it reacts slowly and may not be saturable without complicating side reactions.

Although Prxs are enzymes that catalyse the breakdown of peroxides, they are unusual in that they operate under conditions that are atypical for most enzymes. Most enzymes function at concentrations well below those of their substrates, they are saturable and their kinetic data are interpreted in these terms. This may be the case for Prxs in the test tube, but not in their cellular environment. Prxs are abundant proteins that are almost always present in considerable excess over their substrates. Concentrations typically decrease by a factor of 10 in the order Prx > thioredoxin > thioredoxin reductase, and except when non-physiological concentrations are added exogenously, intracellular $\mathrm{H}_{2} \mathrm{O}_{2}$ will be sub-micromolar and several orders of magnitude less than the Prx concentration. This is an important consideration when interpreting kinetic data in a physiological setting.

\section{PEROXIDASE ASSAYS}

The most straightforward way of assessing whether a protein such as a Prx can break down a peroxide enzymatically is to carry out a peroxidase assay. This can be performed by measuring the rate of peroxide consumption or the rate of oxidation of a reducing substrate such as NADPH in a coupled assay. Such assays have been commonly performed since the initial characterization of Prxs (Chae et al., 1999), using thioredoxin/thioredoxin reductase or an alternative reductant such as DTT. They give useful information on whether a protein has activity, but it should be noted that the reduction step is generally limiting and the turnover rate does not reflect the high peroxide reactivity of the Prx.

A frequent feature of peroxidase assays is that they show a gradual decline in activity over time, even though substrate concentrations do not become limiting (Manta et al., 2009; Yang et al., 2002). This behavior led to the recognition that Prxs could be inactivated by excess peroxide and that eukaryotic Prxs are generally more prone to this hyperoxidation than their prokaryotic counterparts (Wood et al., 2003). By following the time course of peroxidase activity, it is possible to obtain a measure of susceptibility to inactivation (Nelson et al., 2013). As expected for competition between hyperoxidation and condensation of the sulfenic acid with the resolving Cys (discussed above), peroxidase activity is lost sooner at higher peroxide concentrations. To account for this, it has been proposed (Nelson et al., 2013) that the peroxide concentration at which hyperoxidation occurs at 1 in every 100 turnovers be used as a quantitative measure for comparing the susceptibility of individ- 
ual Prxs to inactivation. This is a similar concept to the $k_{2} / k_{3}$ ratio obtained by the competitive method (above) and they report $60 \mu \mathrm{M}$ for Prx1 compared with $>20 \mathrm{mM}$ for some of the bacterial Prxs, illustrating their differing sensitivities.

\section{CONCLUSIONS}

Kinetic data have been important for characterizing the reactivity of Prxs with different substrates, distinguishing oxidation from hyperoxidation and identifying structural features than determine reactivity. Individual Prxs have been shown to exhibit different kinetic properties, although there are still many family members that would benefit from more extensive kinetic analysis. Different methods can be used and often give independent validation or complementary information. However, quantitative rate constant measurements are most informative and applicable to both molecular and cellular studies.

\section{ACKNOWLEDGMENTS}

The authors' work on which this article is based has been supported by the Health Research Council of New Zealand.

\section{REFERENCES}

Chae, H.Z., Kim, H.J., Kang, S.W., and Rhee, S.G. (1999). Characterization of three isoforms of mammalian peroxiredoxin that reduce peroxides in the presence of thioredoxin. Diabetes Res. Clin. Pract. 45, 101-112.

Cox, A.G., Pearson, A.G., Pullar, J.M., Jonsson, T.J., Lowther, W.T., Winterbourn, C., and Hampton, M.B. (2009a). Mitochondrial peroxiredoxin 3 is more resilient to hyperoxidation than cytoplasmic peroxiredoxins. Biochem. J. 421, 51-58.

Cox, A.G., Peskin, A.V., Paton, L.N., Winterbourn, C.C., and Hampton, M.B. (2009b). Redox potential and peroxide reactivity of human peroxiredoxin 3. Biochemistry 48, 6495-6501.

Hall, A., Parsonage, D., Poole, L.B., and Karplus, P.A. (2010). Structural evidence that peroxiredoxin catalytic power is based on transition-state stabilization. J. Mol. Biol. 402, 194-209.

Hall, A., Nelson, K., Poole, L.B., and Karplus, P.A. (2011). Structurebased insights into the catalytic power and conformational dexterity of peroxiredoxins. Antioxid. Redox Signal. 15, 795-815.

Hugo, M., Turell, L., Manta, B., Botti, H., Monteiro, G., Netto, L.E., Alvarez, B., Radi, R., and Trujillo, M. (2009). Thiol and sulfenic acid oxidation of AhpE, the one-cysteine peroxiredoxin from Mycobacterium tuberculosis: kinetics, acidity constants, and conformational dynamics. Biochemistry 48, 9416-9426.

Karplus, P.A. (2015). A primer on peroxiredoxin biochemistry. Free Radic. Biol. Med. 80, 183-190.

Manta, B., Hugo, M., Ortiz, C., Ferrer-Sueta, G., Trujillo, M., and Denicola, A. (2009). The peroxidase and peroxynitrite reductase activity of human erythrocyte peroxiredoxin 2. Arch. Biochem. Biophys. 484, 146-154.

Nagy, P., Karton, A., Betz, A., Peskin, A.V., Pace, P., O'Reilly, R.J., Hampton, M.B., Radom, L., and Winterbourn, C.C. (2011). Model for the exceptional reactivity of peroxiredoxins 2 and 3 with hydrogen peroxide: a kinetic and computational study. J. Biol. Chem. 286, 18048-18055.

Nakamura, T., Kado, Y., Yamaguchi, T., Matsumura, H., Ishikawa, K., and Inoue, T. (2010). Crystal structure of peroxiredoxin from Aeropyrum pernix $\mathrm{K} 1$ complexed with its substrate, hydrogen peroxide. J. Biochem. 147, 109-115.

Nelson, K.J., and Parsonage, D. (2011). Measurement of peroxiredoxin activity. Curr. Protoc. Toxicol. Chapter 7, Unit7 10.

Nelson, K.J., Parsonage, D., Karplus, P.A., and Poole, L.B. (2013) Evaluating peroxiredoxin sensitivity toward inactivation by peroxide substrates. Methods Enzymol. 527, 21-40.

Ogusucu, R., Rettori, D., Munhoz, D.C., Netto, L.E., and Augusto, O. (2007). Reactions of yeast thioredoxin peroxidases I and II with hydrogen peroxide and peroxynitrite: rate constants by competitive kinetics. Free Radic. Biol. Med. 42, 326-334.

Parsonage, D., Youngblood, D.S., Sarma, G.N., Wood, Z.A., Karplus, P.A., and Poole, L.B. (2005). Analysis of the link between enzymatic activity and oligomeric state in AhpC, a bacterial peroxiredoxin. Biochemistry 44, 10583-10592.

Parsonage, D., Nelson, K.J., Ferrer-Sueta, G., Alley, S., Karplus, P.A., Furdui, C.M., and Poole, L.B. (2015). Dissecting peroxiredoxin catalysis: separating binding, peroxidation, and resolution for a bacterial AhpC. Biochemistry 54, 1567-1575.

Peskin, A.V., Low, F.M., Paton, L.N., Maghzal, G.J., Hampton, M.B., and Winterbourn, C.C. (2007). The high reactivity of peroxiredoxin 2 with $\mathrm{H}(2) \mathrm{O}(2)$ is not reflected in its reaction with other oxidants and thiol reagents. J. Biol. Chem. 282, 11885-11892.

Peskin, A.V., Cox, A.G., Nagy, P., Morgan, P.E., Hampton, M.B. Davies, M.J., and Winterbourn, C.C. (2010). Removal of amino acid, peptide and protein hydroperoxides by reaction with peroxiredoxins 2 and 3. Biochem. J. 432, 313-321.

Peskin, A.V., Dickerhof, N., Poynton, R.A., Paton, L.N., Pace, P.E., Hampton, M.B., and Winterbourn, C.C. (2013). Hyperoxidation of Peroxiredoxins 2 and 3: Rate constants for the reactions of the sulfenic acid of the peroxidative cysteine. J. Biol. Chem. 288 , $14170-14177$.

Peskin, A.V., Pace, P.E., Behring, J.B., Paton, L.N., Soethoudt, M., Bachschmid, M.M., and Winterbourn, C.C. (2016). Glutathionylation of the active site cysteines of peroxiredoxin 2 and recycling by glutaredoxin. J. Biol. Chem. in press.

Rhee, S.G., Chae, H.Z., and Kim, K. (2005). Peroxiredoxins: a historical overview and speculative preview of novel mechanisms and emerging concepts in cell signaling. Free Radic. Biol. Med. 38, 1543-1552.

Trujillo, M., Clippe, A., Manta, B., Ferrer-Sueta, G., Smeets, A., Declercq, J.P., Knoops, B., and Radi, R. (2007). Pre-steady state kinetic characterization of human peroxiredoxin 5 : taking advantage of Trp84 fluorescence increase upon oxidation. Arch. Biochem. Biophys. 467, 95-106.

Trujillo, M., Ferrer-Sueta, G., and Radi, R. (2008). Kinetic studies on peroxynitrite reduction by peroxiredoxins. Methods Enzymol. 441, 173-196.

Winterbourn, C.C., and Hampton, M.B. (2008). Thiol chemistry and specificity in redox signaling. Free Radic. Biol. Med. 45, 549-561.

Winterbourn, C.C., and Hampton, M.B. (2015) Redox biology: signaling via a peroxiredoxin sensor. Nat. Chem. Biol. 11, 5-6.

Wood, Z.A., Poole, L.B., and Karplus, P.A. (2003). Peroxiredoxin evolution and the regulation of hydrogen peroxide signaling Science 300, 650-653.

Yang, K.S., Kang, S.W., Woo, H.A., Hwang, S.C., Chae, H.Z., Kim, K., and Rhee, S.G. (2002). Inactivation of human peroxiredoxin I during catalysis as the result of the oxidation of the catalytic site cysteine to cysteine-sulfinic acid. J. Biol. Chem. 277, 3802938036 . 